\title{
Clinical characteristics of acute pancreatitis in pregnancy: experience based on 121 cases
}

\author{
Lingyu Luo ${ }^{1} \cdot$ Hao Zen ${ }^{1} \cdot$ Hongrong Xu ${ }^{1} \cdot$ Yin Zhu $^{1} \cdot$ Pi Liu $^{1} \cdot$ Liang Xia $^{1} \cdot$ Wenhua He ${ }^{1} \cdot$ Nonghua Lv $^{1}$ (1)
}

Received: 28 June 2017 / Accepted: 28 September 2017 / Published online: 21 November 2017

(c) The Author(s) 2017. This article is an open access publication

\begin{abstract}
Purpose Acute pancreatitis in pregnancy (APIP) is a rare condition; however, it markedly affects maternal and fetal health. This study aimed to describe the types, clinical characteristics, mortality, and the safety and necessity of gestation termination of acute pancreatitis in pregnancy (APIP).

Methods We retrospectively reviewed 121 APIP cases in the Gastroenterology Department of The First Affiliated Hospital of Nanchang University. APIP diagnosis were based on 2012 Atlanta Criteria. The correlation between APIP types, severity, biochemical parameters and mortality was analyzed.

Results The most common symptoms for APIP were abdominal pain (86.8\%) and vomiting (73.6\%). The most common causes for APIP were gallstone (36.4\%) and hypertriglyceridemia (32.2\%) and hypertriglyceridemic APIP was correlated with a higher rate for local complication $(P=0.012)$. Serum calcium level was negatively correlated with the severity of APIP $(P<0.01)$. The overall maternal and fetal mortality rate were $3.3 \%(4 / 121)$ and $11.6 \%(14 / 121)$, respectively. The severity of APIP was significantly correlated with higher risks for maternal and fetal death $(P<0.01) .72 .7 \%$ of moderate-to-severe APIP patients underwent Cesarean section to terminate gestation safely.

Conclusion The most common causes of APIP were gallstone and hypertriglyceridemia. Lower level of serum calcium could be used as an indicator for the severity of the APIP. The severity of APIP was associated with higher risk for neonate asphyxia, and maternal and fetal death.
\end{abstract}

Keywords Acute pancreatitis in pregnancy $\cdot$ Clinical characterization $\cdot$ Maternal and fetal mortality

\section{Introduction}

Acute pancreatitis in pregnancy (APIP) is a rare condition with an approximate incidence of 1 over 1000-12,000 pregnant women [1-4]. The acute onset and difficulty in diagnosis and treatment of APIP significantly threaten the maternal and fetal health $[5,6]$. It was previously demonstrated that the mortality rate was approximately $37 \%$ for the mother and $60 \%$ for the fetus, whereas more recently the numbers have decreased significantly due to the improvements in the diagnosis technique, intensive and neonatal care [2, 7-9].

To date, most paper summarizing the clinical characteristics of APIP were of small sample size. A number of

Nonghua Lv

lunonghua@163.com; lunonghua@ncu.edu.cn

Department of Gastroenterology, The First Affiliated Hospital of Nanchang University, No.17 Yongwai Zheng

Street, Nanchang 330006, China factors have been acknowledged as the pathogenic cause for APIP, among which gallstone remains to be mostly common [10]. Other risk factors were also proposed such as increased maternal age, increased pregnancy number, high fat diet as well as higher body mass index [11]. The diagnosis of APIP is often complicated by other obstetrical emergencies. Therefore, it is crucial for understanding the clinical characteristics of APIP. However, as to the diagnosis and treatment, the specific guideline of APIP is still absent, the major reason probably is related to low incidence rate and scarce clinical data.

In this study, we retrospectively reviewed 121 cases of APIP from 2005 to 2015 in our center to describe the types, clinical characteristics, mortality, and the safety and necessity of gestation termination of acute pancreatitis in pregnancy (APIP) i. 


\section{Materials and methods}

\section{Patients}

This study recruited pancreatitis patients from the previously established pancreatitis database from 2005. Pregnant patients from September 2005 to July 2015 were included in this study. Inclusion criteria were acute pancreatitis diagnosed during pregnancy.

\section{APIP diagnosis and definition}

The acute pancreatitis in pregnancy diagnosis was fulfilled based on Atlanta criteria [12] when at least two of the following: (1) acute upper abdominal pain radiated to the back; (2) serum amylase or lipase level was three times higher than normal; (3) radiological evidence indicates acute pancreatitis. APIP was also divided into three severity categories. Mild acute pancreatitis (MAP) referred to pancreatitis without organ dysfunction or generalized complications. Moderate to severe pancreatitis (MSAP) referred to pancreatitis with transient organ dysfunction or localized/generalized complication within $48 \mathrm{~h}$ after treatment. Severe pancreatitis (SAP) referred to pancreatitis with persistent organ dysfunction or localized/generalized complication for more than $48 \mathrm{~h}$ after treatment. Organ dysfunction was defined based on the modified Marshall scoring [12]. The localized complication referred to acute peri-pancreatic fluid collection (AFPC), pancreatic pseudocyst, acute necrosis and encapsulated necrosis [12]. The generalized complication referred to two of the following: body temperature $>38^{\circ} \mathrm{C}$ or $<36{ }^{\circ} \mathrm{C}$; white blood cell count $>12,000 / \mathrm{mm}^{3}$ or $<4000 /$ $\mathrm{mm}^{3}$; heart rate $>90 \mathrm{bpm}$, respiratory rate $>20 / \mathrm{min}$ or $\mathrm{PCO}_{2}<32 \mathrm{mmHg}$.

APIP was also categorized by different pathogenic causes. Acute gallstone pancreatitis was diagnosed by an increased ALT level > $150 \mathrm{U} / \mathrm{L}$ within $48 \mathrm{~h}$ of onset, as well as radiological evidence of abdominal ultrasonography and magnetic resonance cholangiopancreatography (MRCP) [13]. Hypertriglyceridemic pancreatitis (HTGP) was diagnosed based on Chinese guidelines for the management of acute pancreatitis (Shanghai, 2013) [14] with either a serum triglyceride $\geq 11.3 \mathrm{mmol} / \mathrm{L}$ or serum triglyceride was between 5.65 and $11.3 \mathrm{mmol} / \mathrm{L}$ with a lipid turbidity appearance after excluding gallstone, alcohol or medication factors. Idiopathic pancreatitis was diagnosed with radiological evidence of pancreatitis after excluding gallstone, alcohol, hypertriglyceridemia, medication, trauma, autoimmune and surgical factors [13].

\section{Termination of pregnancy}

Pregnancy was determined to be terminated by experienced obstetricians and gastroenterologists. The indications were confirmed fetal death in utero, obligation to use fetal-toxic medication for pancreatitis or organ failure. All termination was consent by the patient after comprehensive evaluation of surgical risks. The approaches for pregnancy termination included Cesarean section, natural birth including preterm and termed birth, and natural or drug induced abortion.

\section{Laboratory examination and definition}

Complete blood count was examined by automatic analyzer (SySMex XN2000, Sysmex Corporation, China). Blood glucose, lipids, and electrolytes were performed by an automatic biochemical analyzer (Hitachi 7600, Hitachi Limited, Japan). Serum fasting glucose was examined by glucose oxidase method. Serum electrolytes including potassium, sodium and chloride were examined by the indirect ion selective electrode method and calcium was tested by Arsenazo III method. Serum triglyceride was examined by the glycerol phosphate oxidase peroxidase method. Hyperglycemia was defined as a fasting glucose $\geq 7.8 \mathrm{mmol} / \mathrm{L}$ and hypertriglyceridemia was defined as a fasting serum triglyceride $\geq 11.3 \mathrm{mmol} / \mathrm{L}$. Hypocalcaemia was defined as serum calcium $<1.75 \mathrm{mmol} / \mathrm{L}$ and an increased white blood cell count was defined as a white blood cells count $>1 \times 10^{10} / \mathrm{L}$.

\section{Statistical analysis}

All data were analyzed by SPSS 17.0 (SPSS Statistics, Chicago, IL, USA). Quantitative data were compared by $t$ test, whereas categorical data were compared by $\chi^{2}$ Chi square test. Data were compared by non-parametrical test if it was not distributed normally. $P$ value $<0.05$ was considered statistically significant.

\section{Results}

\section{APIP patient characteristics}

Patient characteristics are presented in Table 1 and the pathogenic causes for APIP are shown in Fig. 1. The average age of our patients was $27.8 \pm 5.4$ years. Most patients were at gestational week $24-40$ at the time of APIP diagnosis $(67.8 \%, 82 / 121)$, whereas we noted that 37 patients 
Table 1 APIP patients characteristics by different pathogenic types
Fig. 1 Patient proportion by different causes of APIP. HTGP: hypertriglyceridemic pancreatitis

\begin{tabular}{|c|c|c|c|c|}
\hline & Gallstone & HTGP & Idiopathic & $P$ value \\
\hline Number & 44 & 39 & 32 & \\
\hline Age* & $28 \pm 5.3$ & $28.7 \pm 5.4$ & $26.8 \pm 5.8$ & 0.331 \\
\hline Gravidity* & $2.0 \pm 1.0$ & $2.3 \pm 1.3$ & $2.5 \pm 2.0$ & 0.85 \\
\hline Parity* & $1 \pm 1$ & $0.9 \pm 0.9$ & $1.0 \pm 1.1$ & 0.16 \\
\hline \multicolumn{5}{|l|}{ Trimester of pregnancy on admission, $n(\%)$} \\
\hline Early (1-12 weeks) & $1(2.3)$ & $0(0)$ & $1(3.1)$ & \multirow[t]{3}{*}{0.88} \\
\hline Mid (12-24 weeks) & $16(36.4)$ & $15(38.5)$ & $11(34.4)$ & \\
\hline Late (24-40 weeks) & $27(61.4)$ & $24(61.5)$ & $20(62.5)$ & \\
\hline Gestational weeks on admission & $29.7 \pm 6.9$ & $28.6 \pm 7.7$ & $33.7 \pm 4.5$ & 0.06 \\
\hline Interval between diagnosis and delivery & $1.8 \pm 1.3$ & $1.8 \pm 1.3$ & $2.3 \pm 1.8$ & 0.09 \\
\hline \multicolumn{5}{|l|}{ Severity of APIP*, $n(\%)$} \\
\hline MAP & $24(54.5)$ & $12(30.8)$ & $18(56.3)$ & \multirow[t]{3}{*}{0.091} \\
\hline MSAP & $15(34.1)$ & $18(46.2)$ & $12(37.5)$ & \\
\hline SAP & $5(11.4)$ & $9(23.1)$ & $2(6.2)$ & \\
\hline \multicolumn{5}{|l|}{ Localized complications, $n(\%)$} \\
\hline APFC & $13(29.5)$ & $20(51.3)$ & $6(18.8)$ & \multirow[t]{4}{*}{0.012} \\
\hline Pancreatic pseudocyst & $1(2.3)$ & $3(7.7)$ & $1(3.1)$ & \\
\hline Acute necrosis & $1(2.3)$ & $3(7.7)$ & $1(3.1)$ & \\
\hline Encapsulated necrosis & $1(2.3)$ & $3(7.7)$ & $0(0)$ & \\
\hline \multicolumn{5}{|l|}{ Organ dysfunction, $n(\%)$} \\
\hline Respiratory & $13(29.5)$ & $14(35.9)$ & $9(28.1)$ & \multirow[t]{6}{*}{0.742} \\
\hline Circulation & $1(2.3)$ & $2(5.1)$ & $1(3.1)$ & \\
\hline Kidney & $2(4.6)$ & $4(10.3)$ & $1(3.1)$ & \\
\hline Multiple organ dysfunction & $3(6.8)$ & $4(10.3)$ & $1(3.1)$ & \\
\hline History of hypertension, $n(\%)$ & $0(0)$ & $1(2.6)$ & $1(3.1)$ & \\
\hline History of diabetes, $n(\%)$ & $0(0)$ & $6(15.4)$ & $1(3.1)$ & \\
\hline Fatty liver disease, $n(\%)$ & $5(11.4)$ & $7(17.9)$ & $3(9.4)$ & 0.518 \\
\hline Ascite, $n(\%)$ & 17 (38.6) & $20(51.3)$ & $9(28.1)$ & 0.137 \\
\hline Pleural effusion, $n(\%)$ & $20(45.5)$ & $27(69.2)$ & $17(53.1)$ & 0.131 \\
\hline
\end{tabular}

Quantitative data were presented as number and percentage and compared by $\chi^{2}$ Chi square test $A P I P$ acute pancreatitis in pregnancy, $H T G P$ hypertriglyceridemic pancreatitis, $M A P$ mild acute pancreatitis, $M S A P$ moderate to severe acute pancreatitis, $S A P$ severe acute pancreatitis, $A P F C$ acute peri-pancreatic fluid collection

*Data were compared using non-parametrical test

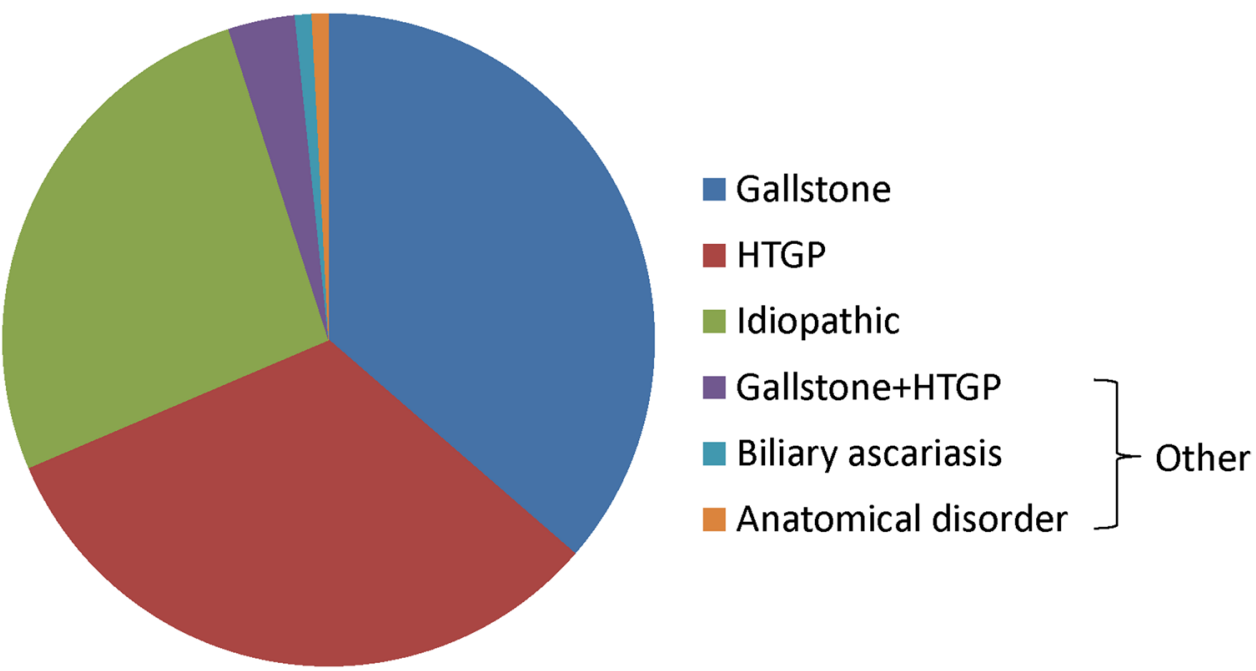


had APIP during the second trimester and 2 patients during the first trimester. The main causes for APIP were gallstone (36.4\%), hypertriglyceridemia (32.2\%) and idiopathic pancreatitis (26.4\%) (Fig. 1). Other causes of APIP included biliary ascariasis (1 case), anatomical disorder (1 case) and gallstone complicated with hypertriglyceridemia (4 cases) (Fig. 1). 49\% (59) were mild acute pancreatitis (MAP), 36\% (44) moderate to severe acute pancreatitis (MSAP) and 15\% (18) were severe acute pancreatitis (SAP).

We further compared clinical characteristics among three major pathogenic causes. The severity of APIP was not significantly different among causes of APIP $(P=0.09)$ (Table 1). When comparing the pancreatitis complications, the hypertriglyceridemia APIP had the highest proportion of acute peri-pancreatic fluid collection $(P=0.012)$. There is no difference between other pancreatitis complications, gestational characteristics, and organ dysfunction or diseases history among different causes (Table 1).

\section{Clinical manifestations of APIP}

In this study, we found that abdominal pain and vomit remained the two most predominant clinical symptoms in our patients. The location of abdominal pain was mainly in the upper abdomen, which occurred in $86.8 \%$ patients $(105 / 121)$ whereas only $11.6 \%$ had lower $(5.0 \%, 6 / 121)$ or generalized abdominal pain $(6.6 \%, 8 / 121)$. More than half of the patients had vomit $(73.6 \%, 89 / 121)$ and fever was less common $(23.1 \%, 28 / 121)$. The major finding after physical examination included abdominal tenderness $(81.8 \%, 99 / 121)$ and rebound tenderness $(34.7 \%, 42 / 121)$. The location of abdominal tenderness was mainly at the upper abdomen $(91 \%, 90 / 99)$, whereas only $3 \%$ occurred at the lower abdomen (3/99) and 6\% at the whole abdomen $(6 / 99)$.

Table 2 Severity of APIP and abnormality of serum examination

\begin{tabular}{llllc}
\hline & MAP & MSAP & SAP & $P$ value \\
\hline Number & 59 & 44 & 18 & \\
Hyperglycemia & $10(16.9)$ & $13(29.5)$ & $7(38.9)$ & 0.111 \\
Hypertriglyceridemia & $10(16.9)$ & $13(29.5)$ & $7(38.9)$ & 0.185 \\
hypocalcaemia & $3(5.1)$ & $7(15.9)$ & $8(44.4)$ & $<0.01$ \\
$\begin{array}{l}\text { Increased white blood cell } \\
\quad \text { count }\end{array}$ & $45(76.3)$ & $36(81.8)$ & $15(83.3)$ & 0.712 \\
\hline
\end{tabular}

Data were presented as number (percentage) and compared by Chi square test; hyperglycemia was defined as fasting glucose $\geq 7.8 \mathrm{mmol} / \mathrm{L}$; hypocalcaemia was defined as serum calcium $<1.75 \mathrm{mmol} / \mathrm{L}$; hypertriglyceridemia was defined as serum triglyceride $\geq 11.3 \mathrm{mmol} / \mathrm{L}$; increased white blood cell count was defined as white blood cells $>1 \times 10^{10} / \mathrm{L}$

$M A P$ mild acute pancreatitis, $M S A P$ moderate to severe acute pancreatitis, $S A P$ severe acute pancreatitis

\section{Laboratory abnormality and severity of APIP}

Some commonly used laboratory results were compared among MAP, MASP and SAP based on the severity (Table 2). Only the level of serum calcium was negatively correlated with the severity of APIP $(P<0.01)$. However, serum glucose, triglycerides, or white blood cell count was not correlated with the severity of APIP (Table 2).

\section{Maternal and fetal outcomes}

\section{Maternal and fetal mortality}

In our study, there were 4 cases of maternal death (with fetal death) in total (3.3\%). One was due to cardiac sudden death (HTGP, at week 13) and 3 were due to multiple organ dysfunctions (1 HTGP, at week 37; the other 2 cases were biliary pancreatitis, at week 38 and 13 , respectively). Besides the 4 cases of maternal with fetal death, another 10 cases of fetal death occurred (Table 3), among which $5.1 \%$ of fetal death occurred in the MAP group, $6.8 \%$ in MASP, and $44.4 \%$ in the SAP group. The mortality rate was

Table 3 Severity, pathogenic types of APIP and fetal mortality

\begin{tabular}{llll}
\hline & Living & Dead & $P$ value \\
\hline $\begin{array}{l}\text { Number } \\
\text { Severity of APIP, } n(\%)\end{array}$ & 107 & 14 & \\
MAP & $56(52.3)$ & $3(21.4)$ & $<0.01$ \\
MSAP & $41(38.3)$ & $3(21.4)$ & \\
SAP & $10(9.3)$ & $8(57.1)$ & \\
Pathogenesis of APIP, & $n(\%)$ & & 0.08 \\
Gallstone & $39(36.4)$ & $5(35.7)$ & \\
HTGP & $31(29.0)$ & $8(57.1)$ & \\
Idiopathic & $31(29.0)$ & $1(7.1)$ & \\
\hline
\end{tabular}

Data were presented as number (percentage) and compared by Chisquare test

$A P I P$ acute pancreatitis in pregnancy, MAP mild acute pancreatitis, $M S A P$ moderate to severe acute pancreatitis, SAP severe acute pancreatitis, $H T G P$ hypertriglyceridemic pancreatitis

Table 4 Apgar score of living neonates delivered by Cesarean birth $(N=48)$

\begin{tabular}{llrrr}
\hline & \multicolumn{2}{l}{ Apgar score } & & $P$ value \\
\cline { 2 - 4 } & $0-3$ & $4-7$ & $8-10$ & \\
\hline MAP & 0 & 3 & 14 & $<0.01$ \\
MSAP & 1 & 10 & 15 & \\
SAP & 2 & 1 & 2 & \\
\hline
\end{tabular}

Data were presented as number by each category

$M A P$ mild acute pancreatitis, $M S A P$ moderate to severe acute pancreatitis, $S A P$ severe acute pancreatitis 
positively correlated with the severity of APIP $(P<0.01)$. However, it was not significantly different among different pathogenic causes of APIP $(P=0.08$, Table 4$)$.

Due to pancreatitis Apgar scores at 5 min of the neonates were evaluated in 48 live Cesarean births (Table 4). It was demonstrated that asphyxia in the neonates was significantly correlated with the severity of APIP of the mother $(P<0.01)$.

The maternal and fetal mortality is recorded in Table 5. Among 121 patients, Gestation was terminated by different approaches, mainly based on fetal and maternal conditions.

\section{Discussion}

This case series study described 121 cases of acute pancreatitis in pregnancy in Chinese and showed that the severity of the APIP was correlated with risk for neonate asphyxia, as well as maternal and fetal death. To our knowledge, this is so far the largest study focusing on acute pancreatitis in pregnancy.

Our data confirmed that the most common causes for acute pancreatitis in pregnancy were gallstone and hypertriglyceridemia in Chinese. Alcohol was considered rare in Chinese maternal population due to a relatively lower rate for Chinese women who regularly takes alcohol drinks [15]. Idiopathic APIP also accounted for $26 \%$ in our population. Although we have ruled out other possible causes before the diagnosis of idiopathic APIP, it remained possible that some of the idiopathic APIP was partially due to high triglyceride level which had dropped after a few days of fasting. Our data also demonstrated that half of the HTGP had acute peri-pancreatic fluid collection. Similarly, previous studies also pointed out that HTGP is more threatening than other

Table 5 Maternal and fetal mortality by different APIP severity

\begin{tabular}{llll}
\hline & MAP & MSAP & SAP \\
\hline Total number & 59 & 44 & 18 \\
Total life birth, $n(\%)$ & 55 & 40 & 10 \\
Continued pregnancy & 35 & 8 & 3 \\
Live Cesarean birth & $18(30.5)$ & $32(72.7)$ & $6(33.3)$ \\
Termed birth & $2(3.4)$ & 0 & $1(5.6)$ \\
Total dead birth, $n(\%)$ & 4 & 4 & 8 \\
Dead Cesarean birth & 0 & $2(4.5)$ & $2(11.1)$ \\
Preterm birth & $1(1.7)$ & $1(2.3)$ & 0 \\
Drug induction labor & $2(3.4)$ & $1(2.3)$ & $2(11.1)$ \\
Natural abortion & $1(1.7)$ & 0 & 0 \\
Maternal and fetal death & 0 & 0 & $4(22.2)$ \\
\hline
\end{tabular}

$A P I P$ acute pancreatitis in pregnancy, MAP mild acute pancreatitis, $M S A P$ moderate to severe acute pancreatitis, $S A P$ severe acute pancreatitis types of APIP [16, 17]. These data indicated that HTGP was associated with higher risk for localized complications.

In our study, most patients had APIP onset during the third trimester (68\%) but we also had 2 and $31 \%$ had APIP during the first and the second trimester. This proportion was similar from previous studies reporting that most APIP occurred in the third trimester [18, 19]. The underlying mechanism is probably due to the compression to the pancreas and gallbladder by the enlarging uterus, as well as changing in steroids that directly affect gallbladder function [20]. Bolukbas et al. also found that even in early pregnancy, a significant drop in gallbladder ejection fraction was demonstrated [21]. Our data also showed the negative relations between serum calcium and the severity of APIP, which is consistent in non-pregnant pancreatitis patients. A testing of serum calcium may indicate the severity of the disease and more studies are warranted to confirm this.

Our results also demonstrated a close relationship between severity of APIP and maternal and fetal outcome. In our study, once there was proof of single or multiple organ failures for the patient, we consulted the obstetric doctors to assess the safety and feasibility of the operation for terminating pregnancy to rescue the lives of APIP patients and the fetus. It could reduce the effect of those facilitating factors due to gestation itself as well as save the fetus as much as possible. A total of 4 maternal deaths (3.3\%) occurred all in the SAP group. SAP group also had the highest rate for fetal death (44\%). In severe pancreatitis, systematic inflammation cytokine explosion could lead to generalized endothelial injury, which cause further tissue damage. A previous study by Sun et al. also indicated that the increased intra-abdominal pressure during SAP was also associated with higher risk of fetal death [22]. On the other hand, it was also noted that $42.9 \%$ of fetal death occurred in the MSP and MASP groups. One possible reason was that severe APIP during early pregnancy is particularly threatening to fetal life due to the severe onset of the disease [23]. Taken together, the severity of APIP determines the risk for neonatal health, and maternal and fetal death.

Termination of pregnancy might be also a key and necessary step for the treatment of APIP. Our data showed that the interval between diagnosis of APIP and delivery were $2.1 \pm 1.3,1.8 \pm 1.4$ and $2.9 \pm 2.3$ days for MAP,MSAP and SAP patients, respectively. For the patients with APIP, organ dysfunction will be assessed on admission at $48 \mathrm{~h}$ after hospitalization, and the termination of pregnancy will be suggested if the following situations exist: (1) when single or multiple organ dysfunction exists, continuing pregnancy might aggravate the disease; (2) during early or midtrimester of pregnancy, clinical medications might affect the growth of the fetus; (3) fetal development is basically mature (after 37 weeks), SIRS caused by AP and clinical medications might increase 
the risk of fetal death. Moreover, we found that MSAP patients underwent the highest rate $(72.7 \%)$ of Cesarean section to get live birth. The reasons might be: (1) most of APIP was occurred in the third trimester and the development of the fetus was relatively mature; (2) the general condition of MASP patients was usually stable enough to complete the operation; (3) Most MASP patients preferred to terminate the pregnancy beforehand to avoid potential harms to the fetus. Fortunately, no maternal death was caused by the operation. The benefits for the patients include preventing the deterioration of APIP and allowing more effective therapy without considering the status of the fetus. So once one or more indications were met, Cesarean section would be a safe and necessary option to stop the deterioration of pancreatitis and save both the mother and the baby.

In conclusion, acute pancreatitis in pregnancy is a rare but severe disease potentially threatening the maternal and fetal life. The most common causes of APIP were gallstone and hypertriglyceridemia, and APIP due to hypertriglyceridemia tends to be associated with more complications. Lower level of serum calcium could be used as an indicator for APIP severity. The severity of the APIP was correlated with higher risk for neonate asphyxia, and maternal and fetal death. Appropriate timing for termination of pregnancy is necessary and safe for APIP patients. More studies are warranted for further elucidation of the etiology, risk factors and treatments of acute pancreatitis in pregnancy, especially the severe type.

Author contributions LYL: Project development, data collection, manuscript writing; HZ:Project development, data collection; HRX: Data collection; YZ: Data collection; PL: Data collection; LX:, Data collection; WHH: Data collection; NHL: Protocol/project development

\section{Compliance with ethical standard}

Ethical approval All procedures performed in studies involving human participants were in accordance with the ethical standards of the First Affiliated Hospital of Nanchang University, Jiangxi Province, China (Reference number: 2011001) and with the 1964 Helsinki declaration and its later amendments or comparable ethical standards.

Informed consent Informed consent was obtained from all individual participants.

Conflict of interest The authors declare that they have no conflict of interest.

Open Access This article is distributed under the terms of the Creative Commons Attribution 4.0 International License (http://creativecommons.org/licenses/by/4.0/), which permits unrestricted use, distribution, and reproduction in any medium, provided you give appropriate credit to the original author(s) and the source, provide a link to the Creative Commons license, and indicate if changes were made.

\section{References}

1. Hernandez A, Petrov MS, Brooks DC, Banks PA, Ashley SW, Tavakkolizadeh A (2007) Acute pancreatitis and pregnancy: a 10-year single center experience. J Gastrointest Surg 11(12):1623-1627. doi:10.1007/s11605-007-0329-2

2. Eddy JJ, Gideonsen MD, Song JY, Grobman WA, O'Halloran P (2008) Pancreatitis in pregnancy. Obstet Gynecol 112(5):10751081. doi:10.1097/AOG.0b013e318185a032 (112/5/1075 [pii])

3. Pitchumoni CS, Yegneswaran B (2009) Acute pancreatitis in pregnancy. World J Gastroenterol 15(45):5641-5646

4. Nanda S, Gupta A, Dora A (2009) Acute pancreatitis: a rare cause of acute abdomen in pregnancy. Arch Gynecol Obstet 279(4):577-578. doi:10.1007/s00404-008-0755-8

5. Montgomery WH, Miller FC (1970) Pancreatitis and pregnancy. Obstet Gynecol 35(4):658-664

6. Ducarme G, Maire F, Chatel P, Luton D, Hammel P (2014) Acute pancreatitis during pregnancy: a review. J Perinatol 34(2):87-94. doi:10.1038/jp.2013.161 (jp2013161 [pii])

7. Geng Y, Li W, Sun L, Tong Z, Li N, Li J (2011) Severe acute pancreatitis during pregnancy: eleven years experience from a surgical intensive care unit. Dig Dis Sci 56(12):3672-3677. doi:10.1007/s10620-011-1809-5

8. Date RS, Kaushal M, Ramesh A (2008) A review of the management of gallstone disease and its complications in pregnancy. Am J Surg 196(4):599-608. doi:10.1016/j.amjsurg.2008.01.015 (S0002-9610(08)00308-5 [pii])

9. Sun L, Li W, Geng Y, Shen B, Li J (2011) Acute pancreatitis in pregnancy. Acta Obstet Gynecol Scand 90(6):671-676. doi:10.1111/j.1600-0412.2011.01072.x

10. Papadakis EP, Sarigianni M, Mikhailidis DP, Mamopoulos A, Karagiannis V (2011) Acute pancreatitis in pregnancy: an overview. Eur J Obstet Gynecol Reprod Biol 159(2):261-266. doi:10.1016/j.ejogrb.2011.07.037

11. Igbinosa O, Poddar S, Pitchumoni C (2013) Pregnancy associated pancreatitis revisited. Clin Res Hepatol Gastroenterol 37(2):177-181. doi:10.1016/j.clinre.2012.07.011 (S2210-7401(12)00215-X [pii])

12. Banks PA, Bollen TL, Dervenis C, Gooszen HG, Johnson CD, Sarr MG, Tsiotos GG, Vege SS (2013) Classification of acute pancreatitis-2012: revision of the Atlanta classification and definitions by international consensus. Gut 62(1):102-111. doi:10.1136/gutjnl-2012-302779 (gutjnl-2012-302779 [pii])

13. IAP/APA (2013) IAP/APA evidence-based guidelines for the management of acute pancreatitis. Pancreatology 13(4 Suppl 2):e1-15. doi:10.1016/j.pan.2013.07.063 (S14243903(13)00525-5 [pii])

14. Pancreas Study Group CSoG, Chinese Medical Association, Editorial Board of Chinese Journal of Pancreatology, Editorial Board of Chinese Journal of Digestion (2013) Chinese guidelines for the management of acute pancreatitis (Shanghai, 2013). J Clin Hepatol 29(9):656-660

15. Millwood IY, Li L, Smith M, Guo Y, Yang L, Bian Z, Lewington S, Whitlock G, Sherliker P, Collins R, Chen J, Peto R, Wang H, Xu J, He J, Yu M, Liu H, Chen Z, China Kadoorie Biobank collaborative g (2013) Alcohol consumption in 0.5 million people from 10 diverse regions of China: prevalence, patterns and socio-demographic and health-related correlates. Int J Epidemiol 42(3):816-827. doi:10.1093/ije/dyt078

16. Abu Musa AA, Usta IM, Rechdan JB, Nassar AH (2006) Recurrent hypertriglyceridemia-induced pancreatitis in pregnancy: a management dilemma. Pancreas 32(2):227-228. doi:10.1097/01. mpa.0000202943.70708.2d (00006676-200603000-00020 [pii])

17. Kayatas SE, Eser M, Cam C, Cogendez E, Guzin K (2010) Acute pancreatitis associated with hypertriglyceridemia: a 
life-threatening complication. Arch Gynecol Obstet 281(3):427429. doi:10.1007/s00404-009-1183-0

18. Vilallonga R, Calero-Lillo A, Charco R, Balsells J (2014) Acute pancreatitis during pregnancy, 7-year experience of a tertiary referral center. Cirugia espanola 92(7):468-471. doi:10.1016/j. ciresp.2013.12.016

19. Mali $P$ (2016) Pancreatitis in pregnancy: etiology, diagnosis, treatment, and outcomes. Hepatobiliary and Pancreat Dis Int: HBPD INT 15(4):434-438

20. Braverman DZ, Johnson ML, Kern F Jr (1980) Effects of pregnancy and contraceptive steroids on gallbladder function. N Engl J Med 302(7):362-364. doi:10.1056/NEJM198002143020702
21. Bolukbas FF, Bolukbas C, Horoz M, Ince AT, Uzunkoy A, Ozturk A, Aka N, Demirci F, Inci E, Ovunc O (2006) Risk factors associated with gallstone and biliary sludge formation during pregnancy. J Gastroenterol Hepatol 21(7):1150-1153. doi:10.1111/j.1440-1746.2006.04444.x

22. Sun L, Li W, Sun F, Geng Y, Tong Z, Li J (2015) Intra-abdominal pressure in third trimester pregnancy complicated by acute pancreatitis: an observational study. BMC Pregnancy Childbirth 15:223. doi:10.1186/s12884-015-0651-8

23. Abdullah B, Kathiresan Pillai T, Cheen LH, Ryan RJ (2015) Severe acute pancreatitis in pregnancy. Case Reports Obstet Gynecol 2015:239068. doi:10.1155/2015/239068 